\title{
Microvolt T-wave alternans in young myocardial infarction patients with preserved cardiac function treated with single-vessel primary percutaneous coronary intervention
}

\author{
Mustafa Umut Somuncu, Mehmet Erturk, Huseyin Karakurt, Ali Riza Demir, Umit Bulut, \\ Asli Kurtar, Ertan Akbay, Ozgur Akgul
}

Mehmet Akif Ersoy Thoracic and Cardiovascular Surgery Center, Training and
Research Hospital, Istanbul, Turkey

Submitted: 25 June 2016

Accepted: 13 July 2016

Arch Med Sci Atheroscler Dis 2016; 1: e68-e74

DOI: 10.5114/amsad.2016.61494

Copyright (C) 2016 Termedia \& Banach

\section{Abstract}

Introduction: Myocardial infarction continues to be the most important cause of morbidity and mortality, and recently this disease has begun to be seen commonly at young ages. In our study we aimed to assess microvolt T-wave alternans in young patients who had ST segment elevation myocardial infarction with preserved left ventricular function and who underwent single-vessel revascularization.

Material and methods: We enrolled 108 consecutive patients (age: 39.5 \pm 4.1 ) with ST segment elevation myocardial infarction (STEMI) undergoing percutaneous coronary intervention and 43 patients (age: $38.5 \pm 3.7$ ) with normal coronary angiograms as a control group. The myocardial infarction patients were younger than 45 and had a preserved left ventricular ejection fraction. They were divided into three groups according to the culprit artery. The microvolt T-wave alternans (MTWA) values were calculated an average of 12 months after the primary percutaneous coronary intervention using the modified moving average method.

Results: The MTWA positivity was significantly higher in the STEMI group compared to the controls $(p<0.001)$. It was also significantly higher in STEMI patients with left anterior descending artery lesions compared to patients with circumflex artery and right coronary artery lesions $(p=0.013)$. Moreover, the culprit artery was independent predictor of MTWA positivity $(p=0.043)$.

Conclusions: In STEMI patients of a young age, MTWA positivity was higher than in healthy individuals, especially when the responsible vessel fed a wider region.

Key words: myocardial infarction, sudden cardiac death, young age, microvolt T wave alternans, preserved left ventricular ejection fraction.

\section{Introduction}

Although the incidence of coronary artery disease increases with age, myocardial infarction (MI) has recently been reported in young patients too [1]. Post-MI patients, especially young individuals, experience cardiovascular events repeatedly throughout their lives, and these events are predominantly the cause of death [2]. Young MI patients generally have single-vessel disease and a preserved left ventricular ejection fraction

\author{
Corresponding author: \\ Mustafa Umut Somuncu \\ Mehmet Akif Ersoy Thoracic \\ and Cardiovascular \\ Surgery Center \\ Training and \\ Research Hospital \\ Istasyon Mah. \\ Turgut Ozal \\ Bulvari No. 11 \\ Kuçukcekmece \\ 34050 Istanbul, Turkey \\ Phone: +90 5323401525 \\ E-mail: usomuncu@gmail. \\ com
}


(LVEF) [3]. Due to their age and the presence of less cardiac damage, these patients are often overlooked by physicians. Thus, such patients remain unaware of their condition and how best to manage it.

Sudden cardiac death (SCD) remains a major problem in public health, especially in patients with a history of coronary artery disease. The only effective therapy for primary prevention of SCD is an implantable cardioverter defibrillator (ICD) [4]. Although research has suggested that low LVEF is an important risk factor for SCD [5], it is also important to stratify the risk of SCD in post-MI patients with a preserved LVEF (EF > 45). Several noninvasive methods have been proposed to improve the selection of patients at risk of SCD, but none are considered suitable for use in clinical decision making [6].

Microvolt T-wave alternans (MTWA) has been proposed as a potential indicator of susceptibility to SCD in patients with coronary artery disease and congestive heart failure [7]. T-wave alternans (TWA) is the beat-to-beat variation in the T-wave amplitude related to spatial and/or temporal variations in ventricular repolarization. Several experimental studies have shown that variations in TWA are associated with increased vulnerability to ventricular arrhythmias [8] and also reported that MTWA is associated with an increased risk of arrhythmic events in post-MI patients, particularly those with a low LVEF, and that it can predict total mortality $[9,10]$. Another study found that a positive MTWA test was associated with arrhythmic events in post$\mathrm{MI}$ patients with a preserved EF and that low-risk populations could be stratified with this method [11]. Although these studies have investigated the prognostic value of TWA in cardiac patients, the prevalence and characteristics of TWA in young MI patients and the relationship between TWA and the lesion site have not been elucidated.

In the current study, we assessed MTWA positivity in young $\mathrm{MI}$ patients according to the lesion site. To ensure population homogeneity, we limited the inclusion criteria only to patients with a single-vessel lesion and $\mathrm{MI}$ with preserved EF. We also investigated the relationship between MTWA positivity and inflammation markers.

\section{Material and methods}

\section{Population}

Between January 2009 and January 2012, 151 subjects who fulfilled the selection criteria were included in this single center study. The study included 108 consecutive patients (mean age \pm SD: $39.5 \pm 4.1$ ) with their first ST segment elevation myocardial infarction (STEMI) undergoing a single-vessel primary percutaneous coronary intervention $(\mathrm{PCl})<12 \mathrm{~h}$ from symptom onset and $43 \mathrm{pa}-$ tients (mean age \pm SD: of $38.5 \pm 3.7$ ) with normal coronary angiograms as a control group. The MI patients were divided into three groups according to the lesion site. The culprit artery was: the left anterior descending artery (LAD) in 56 (51.9\%) patients, the circumflex artery (CX) in 22 (20.3\%) patients, and the right coronary artery (RCA) in 30 (27.8\%) patients. The diagnosis of acute $\mathrm{MI}$ was based on the clinical course and ST segment elevation on an electrocardiogram. All the culprit lesions in the MI patients had TIMI grade 3 flow, and there was no significant stenosis in the other vessels. The patients were followed up approximately 1 year later (8-14 months) and underwent MTWA testing Data were collected on the patient's demographics, medical history, cholesterol level, blood cell count, high-sensitivity C-reactive protein (hs-CRP) level, and treatment. Blood samples were drawn in the morning after an overnight fast. Serum lipids, creatinine, and other biochemical blood measurements were determined using standard laboratory procedures. The total white cell count and neutrophil and lymphocyte counts were recorded, and the neutrophil/lymphocyte $(\mathrm{N} / \mathrm{L})$ ratio was calculated. An echocardiographic study with a biplane modified Simpson's method was performed to assess the patients' LVEFs. Exclusion criteria were as follows: (i) low LVEF (EF < 45); (ii) unstable situation on follow-up, such as reinfarction, restenosis, or rehospitalization; (iii) detection of ischemic sign on an exercise test or myocardial perfusion scintigraphy; (iv) chronic disease, such as chronic renal failure or chronic liver failure; (v) moderate or severe valvular disease; (vi) conditions, including atrial fibrillation, pacemaker rhythm, intraventricular conduction defects, and technical artefacts, that could interfere with the TWA analysis during the exercise stress test (EST); (vii) patients with multivessel disease; (viii) all types of infection that could affect inflammation markers. The study protocol was approved by the local ethics committee, and all patients gave informed consent for participation in the study.

\section{Exercise stress test}

The treadmill EST (CardioSoft GE Healthcare System Milwaukee; version 4.14) was carried out according to a standard symptom/sign-limited Bruce protocol. The MTWA analysis was performed noninvasively during submaximal exercise. The Mason-Likar Modification of the standard 12-lead system was used. Continuous ECG monitoring was digitally recorded at $500 \mathrm{~Hz}$ with the CardioSoft exercise ECG system and analyzed automatically using the modified moving average (MMA) method (CardioSoft GE Healthcare System Milwaukee; version 4.14). Blood pressure was measured at baseline, during peak exercise, and during the last minute of each stage of the exercise. 


\section{Measurement of T-wave alternans}

The TWA was assessed with the time-domain MMA method. In this method, the MMA algorithm divides consecutive ECG waveforms into odd and even groups and simultaneously creates mean templates of these groups. Then average morphologies of both were calculated separately and updated to every new incoming beat by a weighting factor. This minimizes the influence of noise and artefacts, such as those caused by respiration and walking. During the stress test, the maximal difference between the averages of the odd and even beats along one of the J-T segment sample points in any lead was accepted as the TWA value. Periods where the levels were larger than $10 \mu \mathrm{V}$, periods where no T wave could be clearly seen, periods where the TWA values were $>125$ heart rate or periods where the templates of the QRS wave complexes could not be superimposed completely were excluded from the analysis. These processes were repeated until the accepted TWA values were obtained. The TWA values were calculated continuously at all standard leads during the entire exercise test from rest to recovery. The TWA values were recorded numerically ( $T$ num) and categorically (T65). If the value was higher than the cut-off value of 65 , which was based on a previously published report [12], the TWA was considered positive. All the MTWA tests were performed and interpreted by an expert cardiologist who was blinded to the characteristics of the patients.

\section{Statistical analysis}

Statistical analyses were performed using SPSS software version 17.0 for Windows (SPSS Inc., Chicago, Illinois, USA). Visual (histograms, probability plots) and analytical methods (Kolmogorov-Smirnov/Shapiro-Wilk test) were used to determine the normal distribution of the variables. Descriptive analyses are presented as means and standard deviations. The categorical variables are expressed as numbers and percentages. Numerical variables were compared using Student's t-test, a one-way ANOVA test, Kruskal-Wallis test, or Mann-Whitney $U$ test. The Mann-Whitney $U$ test or Tukey's test was used to determine the significance of pairwise differences. A Bonferroni correction was employed to adjust for multiple comparisons. Categorical data were compared with a $\chi^{2}$ test. In addition, forward stepwise multivariate logistic regression models were created to identify the independent correlates of MTWA positivity. Variables with a $p$-value $<0.10$ in the univariate analysis were included in the multivariate model. The statistical significance level was considered to be $p<0.05$.

\section{Results}

\section{General characteristics}

Table I shows the main clinical characteristics of the MI patients and the control group. The prevalence of smoking, hypertension, and hypercholesterolemia (cardiovascular risk factors) was

Table I. Clinical and biochemical characteristics of MI patients and normal group

\begin{tabular}{|lccc|}
\hline Parameter & $\begin{array}{c}\text { MI patients } \\
(n=108)\end{array}$ & $\begin{array}{c}\text { Control group } \\
(n=43)\end{array}$ & $P$-value \\
\hline Age [years] & $39.5 \pm 4.1$ & $38.5 \pm 3.7$ & 0.161 \\
\hline Male, $n(\%)$ & $98(90.6)$ & $34(86)$ & 0.407 \\
\hline BMI $\left[\mathrm{kg} / \mathrm{m}^{2}\right]$ & $29.1 \pm 3.8$ & $28.6 \pm 4.1$ & 0.545 \\
\hline Familiarity for IHD, $n(\%)$ & $34(31.4)$ & $11(25.5)$ & 0.214 \\
\hline Smoking, $n(\%)$ & $83(76.2)$ & $16(37.2)$ & $<0.001$ \\
\hline Diabetes, $n(\%)$ & $13(11.9)$ & $6(14.6)$ & 0.725 \\
\hline Hypertension, $n(\%)$ & $51(43.3)$ & $11(25.6)$ & 0.039 \\
\hline Hyperlipidemia, $n(\%)$ & $58(57.6)$ & $13(30.2)$ & 0.002 \\
\hline Laboratory findings: & & $41.8 \pm 10.2$ & 0.004 \\
\hline HDL [mg/dl] & $36.7 \pm 8.9$ & $119 \pm 18.8$ & 0.007 \\
\hline LDL [mg/dl] & $137.1 \pm 23.3$ & $2.8 \pm 0.8$ & 0.024 \\
\hline hs-CRP [mg/l] & $5.3 \pm 2.1$ & $1.8 \pm 0.5$ & $<0.001$ \\
\hline N/L ratio & $3.7 \pm 1.1$ & $43 \pm 4.1$ & 0.397 \\
\hline Hematocrit & $42 \pm 5.1$ & $62.6 \pm 2.8$ & $<0.001$ \\
\hline LVEF & $53.5 \pm 5.3$ & & \\
\hline
\end{tabular}

$H D L$ - high-density lipoprotein, hs-CRP - high-sensitivity C-reactive protein, IHD - ischemic heart disease, LDL - low-density lipoprotein, $L V E F$ - left ventricular ejection fraction, $N / L$ ratio - neutrophil/lymphocyte ratio. 
Table II. Clinical, biochemical, and medical compliance of MI subgroups

\begin{tabular}{|c|c|c|c|c|}
\hline Parameter & $\begin{array}{l}\text { LAD lesion } \\
\quad(n=56)\end{array}$ & $\begin{array}{l}\text { CX lesion } \\
(n=22)\end{array}$ & $\begin{array}{l}\text { RCA lesion } \\
\quad(n=30)\end{array}$ & $P$-value \\
\hline Age [years] & $39.5 \pm 4.3$ & $39.0 \pm 3.9$ & $39.5 \pm 4.3$ & 0.698 \\
\hline Male, $n(\%)$ & $49(88.1)$ & $20(94.1)$ & $27(90.0)$ & 0.581 \\
\hline Hypertension, $n$ (\%) & $21(38.8)$ & $12(52.8)$ & $13(42.4)$ & 0.397 \\
\hline Hyperlipidemia, n (\%) & $29(56.7)$ & $13(60.6)$ & $16(56.0)$ & 0.919 \\
\hline Diabetes, $n(\%)$ & $7(13.4)$ & $3(11.8)$ & $3(8.8)$ & 0.774 \\
\hline Smoking, $n(\%)$ & $38(71)$ & $17(79.4)$ & $25(84.0)$ & 0.407 \\
\hline LVEF & $52.6 \pm 6.6$ & $54.5 \pm 3.4$ & $54.3 \pm 3.5$ & 0.180 \\
\hline Acetylsalicylic acid & $43(84.8)$ & $19(92)$ & $25(81.4)$ & 0.515 \\
\hline Clopidogrel & $27(48)$ & $8(36)$ & $16(53.1)$ & 0.416 \\
\hline ACE inhibitors & $34(64.2)$ & $12(60.6)$ & $19(63)$ & 0.942 \\
\hline$\beta$-Blockers & $45(80.3)$ & $16(76.1)$ & $23(78.2)$ & 0.898 \\
\hline Statins & $34(64.2)$ & $15(68.2)$ & $18(60.3)$ & 0.902 \\
\hline
\end{tabular}

$A C E$ inhibitor - angiotensin-converting-enzyme inhibitor, LVEF - left ventricular ejection fraction, LAD - left anterior descending artery, $C X$ - circumflex artery, $R C A$ - right coronary artery.

significantly higher in the MI patients compared to the controls. High-density lipoprotein (HDL), low-density lipoprotein (LDL), C-reactive protein (CRP), the N/L ratio, the LVEF were also significantly higher and LVEF was significiantly lower in the MI group than the control group.

Table II presents the characteristics of the MI subgroups (according to the culprit artery). There were no differences in cardiovascular risk factors, age, sex, medical therapy, or EF between the groups. All post-MI patients were also questioned about their medical therapy. Medication compliance was $85.4 \%$ for acetylsalicylic acid (ASA), $62.6 \%$ for angiotensin-converting-enzyme (ACE) inhibitors, $78.9 \%$ for $\beta$-blockers, and $64.2 \%$ for statins. There were no significant differences in medication compliance between the MI groups.
The T num and T65 values of the MI patients were significantly higher compared to those of the control group ( $p<0.001$ and $p=0.007$, respectively) (Table III). In the MI group, 21 (19.4\%) of 108 patients had MTWA positivity. The subgroup analyses revealed a difference in the T num and 65 according to the culprit artery (LAD compared to CX, and RCA) ( $p<0.001$ and $p=0.045$, respectively) (Table III). Sixteen patients with LAD lesions, 3 with RCA lesions, and 2 with CX lesions were MTWA positive. The same significant difference in the T num and T65 values was observed when the groups were divided into LAD and non-LAD $(p<0.001$ and $p=0.013$, respectively) (Table III, Figure 1 ).

Logistic regression analysis was performed to determine independent predictors of MTWA positivity. The LVEF and culprit artery showed statisti-

Table III. Comparisons of MTWA between MI subgroups and control group

\begin{tabular}{|c|c|c|c|c|}
\hline Variable & MI patients $(n=108)$ & \multicolumn{2}{|c|}{ Control group $(n=43)$} & $P$-value \\
\hline T num & 43.3 & \multicolumn{2}{|c|}{14.3} & $<0.001$ \\
\hline $\mathrm{T} 65, n(\%)$ & $21(19.4)$ & \multicolumn{2}{|c|}{$1(2.3)$} & 0.007 \\
\hline Variable & LAD lesion $(n=56)$ & CX lesion $(n=22)$ & RCA lesion $(n=30)$ & $P$-value \\
\hline T num & $52.6 \pm 22.7$ & $30.3 \pm 16.5$ & $36.8 \pm 19.8$ & $<0.001$ \\
\hline T65, $n(\%)$ & $16(28.6)$ & $2(9.1)$ & $3(10)$ & 0.045 \\
\hline Variable & LAD lesion $(n=56)$ & \multicolumn{2}{|c|}{ Non-LAD lesion $(n=52)$} & $P$-value \\
\hline T num & $51.2 \pm 19.7$ & \multicolumn{2}{|c|}{$29.2 \pm 12.2$} & $<0.001$ \\
\hline T65, $n$ (\%) & $16(28.6)$ & \multicolumn{2}{|c|}{$5(9.6)$} & 0.013 \\
\hline
\end{tabular}

LAD - left anterior descending artery, CX - circumflex artery, RCA - right coronary artery, T num - TWA value numerically, T65 - TWA value categorically, $\mathrm{MI}$ - myocardial infarction. 


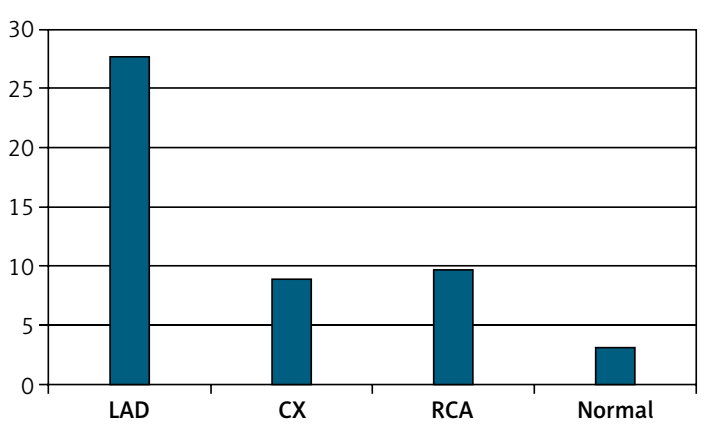

Figure 1. Comparison of MTWA positivity (\%) between MI subgroups and control group $\angle A D$ - left anterior descdending artery, $C X$ - circumflex artery, $R C A$ - right coronary artery.

cal significance in the univariate logistic regression analysis and were incorporated into the multivariate analysis. The results demonstrated that the location of the lesion site was an independent predictor of MTWA positivity (Table IV).

\section{Discussion}

The present study revealed a statistically significant difference in the MTWA values of young $\mathrm{Ml}$ patients with single-vessel revascularization and a preserved EF compared to those of normal coronary angiogram patients. In addition, in the subgroup analysis, the MTWA value was significantly higher in patients with culprit LAD lesions than in patients with RCA and CX culprit lesions. The multivariate analysis identified the lesion site as independent predictor of MTWA. To the best of our knowledge, no previous study has examined MTWA values in young MI patients or the relationship between MTWA positivity and the location of the lesion site.
Most previous studies of TWA have shown that it is a powerful predictor of major arrhythmic events, in particular ischemic heart disease and LV dysfunction $[13,14]$. Hohnloser et al. demonstrated that the TWA test is helpful for detection of sudden cardiac death in low-risk MI patients [14]. Bloomfield et al. reported that an abnormal MTWA test is a strong predictor of all-cause mortality [15]. In another study of 850 post-MI patients, MTWA predicted SCD, with a relative hazard ratio of 11.4 during a 25-month follow-up and a negative predictive value of $99.5 \%$ [16].

Although the EF is an important arrhythmia risk factor and an indicator of ICD treatment, patients with a normal range EF are the most commonly seen group with SCD [17]. Most studies of TWA have included only patients with advanced heart failure [13, 14]. Two included a large population with a preserved EF $[11,16]$. In one of these studies, Ikeda et al. followed 1041 patients for an average of 3 years [11]. They reported that MTWA abnormality was the most important prognostic factor in the detection of life-threatening arrhythmias and SCD, with a hazard ratio of 19.7 In the other study, Exner et al. found that the MTWA test, together with spectral and MMA methods, was a significant prognostic factor in patients with a preserved EF [16]. Based on the findings of the aforementioned studies, which were composed of preserved EF patients, we can speculate that the MTWA positivity in the current prospective study is a prognostic indicator of a poor outcome.

One study in the literature compared MTWA values in MI patients and healthy individuals [18]. In that study, Mollo et al. found that the MI patients had significantly higher MTWA values in

Table IV. Logistic regression analysis: independent predictors of abnormal MTWA in MI patients

\begin{tabular}{|c|c|c|c|c|}
\hline \multirow[t]{2}{*}{ Parameter } & \multicolumn{2}{|c|}{ Univariate analysis } & \multicolumn{2}{|c|}{ Multivariate analysis } \\
\hline & OR $(95 \% \mathrm{Cl})$ & $P$-value & OR $(95 \% \mathrm{Cl})$ & $P$-value \\
\hline Smoking & $0.388(0.124-1.214)$ & 0.104 & & \\
\hline Hematocrit & $1.455(0.556-3.802)$ & 0.445 & & \\
\hline LVEF & $0.904(0.834-0.980)$ & 0.014 & $0.982(0.895-1.077)$ & 0.699 \\
\hline Age & $0.936(0.844-1.038)$ & 0.210 & & \\
\hline Culprit artery & $2.760(1.264-6.027)$ & 0.011 & $3.325(1.040-10.635)$ & 0.043 \\
\hline Sex (male) & $1.315(0.609-2.840)$ & 0.485 & & \\
\hline Creatinine & $4.646(0.331-65.247)$ & 0.255 & & \\
\hline Hypertension & $2.747(0.905-8.335)$ & 0.104 & & \\
\hline Hyperlipidemia & $1.197(0.427-3.335)$ & 0.732 & & \\
\hline Diabetes mellitus & $3.538(0.426-29.32)$ & 0.242 & & \\
\hline WBC & $1.166(1.016-1.388)$ & 0.108 & & \\
\hline
\end{tabular}


a TWA exercise test than patients without a cardiac disease history. In the present study, in parallel with that research, the post-MI patients had higher MTWA values compared to healthy individuals.

One study investigated MTWA values in arterior $\mathrm{MI}$ patients but it did not include the other kind of ST elevated MI [19]. In that study, the percentage of positive MTWA with spectral analysis in anterior $\mathrm{Ml}$ patients was $32.4 \%$. In our study, the percentage was $21.4 \%$ in anterior MI patients. The lower percentage of MTWA positivity in the present study can be explained by the difference in the study populations, with the current one being composed of young patients who had a preserved EF.

In another study, researchers found that early diastolic dysfunction, measured by tissue Doppler imaging, was independently associated with MTWA abnormality in type 2 diabetes mellitus individuals with normal systolic function [20]. The authors assumed that the reduction of E' and $S$ ' might be an indicator of myocardial fibrosis, which leads to cellular decomposition. The resulting diastolic electrical repolarization instability could explain MTWA abnormalities. Although in the present study there was no EF difference in the subgroups, the LAD generally feeds a larger area than the CX and RCA. Therefore, in LAD lesions, the myocardium would show more chronic damage and more fragility. The increased fragility of the tissue may be reflected in greater MTWA positivity.

In the present study, the average TWA values were measured in a 1-year period in post-MI patients. In the study by Ikeda et al., the MTWA assessed 20 days after infarction was highly predictive of survival [21]. In contrast, the MTWA assessed 8 days after $\mathrm{Ml}$ did not predict survival in the study by Tapanainen et al. [22]. The REFINE study compared the prognostic value of the MTWA test 2-4 weeks and 10-14 weeks after infarction and found that it was more valuable in the late period [16]. According to the published data, most researchers do not assess the TWA until at least 6-8 weeks after MI to allow the healing process to complete. Additionally, pharmacological treatment, in particular arrhythmic ones, could be better titrated in the late period. The present study allowed sufficient time to obtain the best TWA results.

Another important issue when evaluating TWA is patient adherence to and compliance with antiarrhythmic drug regimens. Chan et al. demonstrated that the predictive ability of the MTWA test declined dramatically in patients who discontinued $\beta$-blocker therapy prior to the test, reporting a relative risk for $\beta$-blocker users of 5.39 and 1.95 for nonusers [23]. In the current study, there was no break in medication to achieve the most accurate results. It can be speculated that the value of the MTWA test may be improved by conducting the test 3-12 months after infarction to allow sufficient time for healing and for enough drug efficiency. Thus, in the current study, the MTWA test was executed in the late period (approximately 12 months). This approach may be more helpful in determining long-term risks.

First, this was a single-center study with a small but homogeneous population. Second, this study employed the MMA method, which is used less frequently than spectral analysis. However, studies of both the MMA method and spectral analysis have shown a good correlation [17]. In addition, studies have reported that the MMA method is as effective as spectral analysis in the prediction of cardiovascular events [17]. Third, we did not include other noninvasive markers of sudden cardiac death, such as autonomic tonus tests. Fourth, we did not investigate subclinical systolic and diastolic dysfunction with tissue Doppler imaging or spectral analysis, and the myocardial fibrosis which can be related to MTWA positivity was not documented with MRI and scintigraphy or laboratory markers such as galectin. Finally, the present study did not assess the prognostic implications of the findings, because this would require longterm follow-up. This issue needs to be addressed in future studies.

In conclusions, our findings suggest that young MI patients treated with single-vessel percutaneous coronary intervention who have a preserved $E F$, particularly those with LAD lesions, exhibit MTWA positivity. In conclusion, considering the prognostic significance of MTWA positivity, clinicians should be aware that young post-MI patients may experience cardiovascular arrhythmic events, especially if the culprit artery feeds a wider region. Larger studies are needed to examine the reproducibility of these results.

\section{Conflict of interest}

The authors declare no conflict of interest.

\section{References}

1. Zimmermann FH, Cameron A, Fisher LD, Ng G. Myocardial infarction in young adults: angiographic characterization, risk factors and prognosis (Coronary Artery Study Registry). J Am Coll Cardiol 1995; 26: 654-61.

2. Fournier JA, Sanchez A, Quero J, Fernandez-Cortacero JA, Gonzalez-Barrero A. Myocardial infarction in men aged 40 years or less: a prospective clinical angiographic study. Clin Cardiol 1996; 19: 631-6.

3. Hoit BD, Glipin EA, Henning $H$. Myocardial infarction in young patients: an analysis by age subsets. Circulation 1986; 74: 712-21.

4. Zipes DP, Camm AJ, Borggrefe M, Buxton AE, Chaitman B, Fromer M. ACC/AHA/ESC 2006 guidelines for management of patients with ventricular arrhythmias and the 
prevention of sudden cardiac death. Europace 2006; 8 : 746-837.

5. Okutucu S, Oto A. Risk stratification in nonischemic dilated cardiomyopathy: current perspectives. Cardiol 2010; 17: 219-29.

6. Stein KM. Noninvasive risk stratification for sudden death: signal-averaged electrocardiography, nonsustained ventricular tachycardia, heart rate variability, baroreflex sensitivity, and QRS duration. Prog Cardiovasc Dis 2008; 51: 106-17.

7. Gold MR, Ip JH, Costantini O, Poole JE, McNulty S, Mark DB. Role of microvolt T-wave alternans in assessment of arrhythmia vulnerability among patients with heart failure and systolic dysfunction: primary results from the T-wave alternans sudden cardiac death in heart failure trial substudy. Circulation 2008; 118: 2022-8.

8. Cox V, Patel M, Kim J, Liu T, Sivaraman G, Narayan SM. Predicting arrhythmia-free survival using spectral and modified moving average analysis of T-wave alternans. Pacing Clin Electrophysiol 2007; 30: 352-8.

9. Friedman DJ, Bender SR, Markowitz SM, Lerman BB, Okin PM. T wave alternans and ST depression assessment identifies low risk individuals with ischemic cardiomyopathy in the absence of left ventricular hypertrophy. Ann Noninvasive Electrocardiol 2013; 18: 359-68.

10. Ikeda T, Saito H, Tanno K, Shimizu H, Watanabe J, Ohnishi Y. T-wave alternans as a predictor for sudden cardiac death after myocardial infarction. Am J Cardiol 2002; 89: 79-82.

11. Ikeda T, Yoshino $H$, Sugi K. Predictive value of microvolt $\mathrm{T}$ wave alternans for sudden cardiac death in patients with preserved cardiac function after acute myocardial infarction. Am J Cardiol 2006; 48: 2268-74.

12. Nieminen T, Lehtima T, Viik J, Lehtinen R, Nikus K. T-wave alternans predicts mortality in a population undergoing a clinically indicated exercise test. Eur Heart J 2007; 28: 2332-7.

13. Gehi AK, Stein RH, Metz LD, Gomes JA. Microvolt T-wave alternans for the risk stratification of ventricular tachyarrhythmic events: a meta-analysis. J Am Coll Cardiol 2005; 46: 75-82.

14. Hohnloser SH, Ikeda T, Cohen RJ. Evidence regarding clinical use of microvolt T-wave alternans. Heart Rhythm 2009; 6: S36-44.

15. Bloomfield DM, Bigger JT, Steinman RC, Namerow PB, Parides MK, Curtis AB. Microvolt T-wave alternans and the risk of death or sustained ventricular arrhythmias in patients with left ventricular dysfunction. J Am Coll Cardiol 2006; 47: 456-63.

16. Exner DV, Kavanagh KM, Slawnych MP, Mitchell LB, Ramadan D. Refine investigators. Noninvasive risk assessment early after a myocardial infarction the REFINE study. J Am Coll Cardiol 2007; 50: 2275-84.

17. Cox V, Patel M, Kim J, Liu T, Sivaraman G. Predicting arrhythmia-free survival using spectral and modified - moving average analyses of T-wave alternans. Pacing Clin Electrophysiol 2007; 30: 352-8.

18. Mollo R, Cosenza A. T-wave alternans in apparently healthy subjects and in different subsets of patients with ischaemic heart disease. Europace 2012; 14: 272-7.

19. Wita K, Filipecki A. Microvolt T-wave alternans predicts cardiac events after acute myocardial infarction in patients treated with primary percutaneous coronary intervention. Adv Med Sci 2012; 12: 56-60.

20. Bonapace S, Targher G. Relationship between early diastolic dysfunction and abnormal microvolt T-wave alter- nans in patients with type 2 diabetes. Circ Cardiovasc Imaging 2011; 4: 408-14.

21. Ikeda T, Sakata T, Takami M, Kondo N, Tezuka N, Nakae T. Combined assessment of T- wave alternans and late potentials used to predict arrhythmic events after myocardial infarction. A prospective study. J Am Coll Cardiol 2000; 35: 722-30.

22. Tapanainen JM, Still AM, Airaksinen KE, Huikuri HV. Prognostic significance of risk stratifiers of mortality, including T-wave alternans, after acute myocardial infarction: results of a prospective follow-up study. J Cardiovasc Electrophysiol 2001; 12: 645-52.

23. Chan PS, Gold MR, Nallamothu BK. Do beta blockers impact microvolt T-wave alternans testing in patients at risk for ventricular arrhythmias? A meta-analysis. J Cardiovasc Electrophysiol 2010; 21: 1009-14. 